\title{
Column density distribution and cosmological mass density of neutral gas: Sloan Digital Sky Survey-III Data Release 9`
}

\author{
P. Noterdaeme ${ }^{1}$, P. Petitjean ${ }^{1}$, W. C. Carithers ${ }^{2}$, I. Pâris ${ }^{3}$, A. Font-Ribera ${ }^{2,4}$, S. Bailey ${ }^{2}$, E. Aubourg ${ }^{5}$, D. Bizyaev ${ }^{6}$,
} G. Ebelke $^{6}$, H. Finley ${ }^{1}$, J. Ge ${ }^{7}$, E. Malanushenko ${ }^{6}$, V. Malanushenko ${ }^{6}$, J. Miralda-Escudé ${ }^{8}$, A. D. Myers ${ }^{9}$, D. Oravetz ${ }^{6}$, K. Pan ${ }^{6}$, M. M. Pieri ${ }^{10}$, N. P. Ross ${ }^{2}$, D. P. Schneider ${ }^{11,12}$, A. Simmons ${ }^{6}$, and D. G. York ${ }^{13,14}$

1 Institut d'Astrophysique de Paris, CNRS-UPMC, UMR 7095, 98bis bd Arago, 75014 Paris, France e-mail: noterdaeme@iap.fr

2 Lawrence Berkeley National Lab, 1 Cyclotron Rd, Berkeley, CA 94720, USA

3 Departamento de Astronomía, Universidad de Chile, Casilla 36-D, Santiago, Chile

${ }^{4}$ Institute of Theoretical Physics, University of Zurich, 8057 Zurich, Switzerland

5 APC, CNRS UMR 7164, 10 rue Alice Domon et Léonie Duquet, 75205 Paris Cedex 13, France

${ }^{6}$ Apache Point Observatory, PO Box 59, Sunspot, NM 88349-0059, USA

7 Astronomy Department, University of Florida, 211 Bryant Space Science Center, PO Box 112055, Gainesville, FL 32611-2055, USA

8 Institut de Ciències del Cosmos, Universitat de Barcelona, Facultat de Física, Barcelona, Spain

9 Department of Physics and Astronomy, University of Wyoming, Laramie, WY 82071, USA

${ }^{10}$ Institute of Cosmology and Gravitation, University of Portsmouth, UK

11 Department of Astronomy and Astrophysics, The Pennsylvania State University, University Park, PA 16802, USA

12 Institute for Gravitation and the Cosmos, The Pennsylvania State University, University Park, PA 16802, USA

13 Department of Astronomy and Astrophysics, University of Chicago, 5640 South Ellis Avenue, Chicago, IL 60637, USA

14 Enrico Fermi Institute, University of Chicago, 5640 South Ellis Avenue, Chicago, IL 60637, USA

Received 19 August 2012 / Accepted 3 October 2012

\section{ABSTRACT}

\begin{abstract}
We present the first results from an ongoing survey for damped Lyman- $\alpha$ systems (DLAs) in the spectra of $z>2$ quasars observed in the course of the Baryon Oscillation Spectroscopic Survey (BOSS), which is part of the Sloan Digital Sky Survey (SDSS) III. Our full (non-statistical) sample, based on Data Release 9, comprises 12081 systems with $\log N(\mathrm{HI}) \geq 20$, out of which 6839 have $\log N(\mathrm{HI}) \geq 20.3$. This is the largest DLA sample ever compiled, superseding that from SDSS-II by a factor of seven.

Using a statistical sub-sample and estimating systematics from realistic mock data, we probe the $N(\mathrm{HI})$ distribution at $\langle z\rangle=2.5$. Contrary to what is generally believed, the distribution extends beyond $10^{22} \mathrm{~cm}^{-2}$ with a moderate slope of index $\approx-3.5$. This result matches the opacity-corrected distribution observed at $z=0$ surprisingly well. The cosmological mass density of neutral gas in DLAs is found to be $\Omega_{\mathrm{g}}^{\mathrm{DLA}} \approx 10^{-3}$, evolving only mildly over the past 12 billion years.
\end{abstract}

Key words. cosmology: observations - quasars: absorption lines - galaxies: evolution

\section{Introduction}

Studying the distribution of neutral gas in and around galaxies at different cosmological times provides a wealth of information about the formation and evolution of galaxies. The 21-cm hyperfine emission of atomic hydrogen has been used to trace the neutral gas in nearby galaxies and estimate their total Hi mass. Given the sensitivity of current radio telescopes, this technique remains limited to $z \leq 0.2$ (e.g. Lah et al. 2007). At high redshift, neutral gas is revealed by the damped Lyman- $\alpha$ absorption systems (DLAs) that it imprints in the optical spectra of bright background sources such as quasars. Because the detection of DLAs is only cross-section dependent, it is possible to statistically derive the amount of neutral gas and the corresponding column density distribution at different redshifts independently of the nature of the absorbers (see Wolfe et al. 2005).

* Table 3 is only available at the CDS via anonymous ftp to cdsarc.u-strasbg.fr $(130.79 .128 .5)$ or via http://cdsarc.u-strasbg.fr/viz-bin/qcat?]/A+A/547/L1
The most recent contributions to the census of DLAs have used data mining of thousands of quasar spectra from the SDSS (SDSS, York et al. 2000) by Prochaska et al. (2005), Prochaska et al. (2009), and Noterdaeme et al. (2009, hereafter N09). These studies indicate that the $N(\mathrm{HI})$ distribution function $\left(f\left(N_{\mathrm{HI}}, \chi\right)\right.$, where $\chi$ is the absorption distance, see Lanzetta et al. 1991) steepens at $\log N(\mathrm{HI})>21$ and that the cosmological density of neutral gas contained in DLAs $\left(\Omega_{\mathrm{g}}^{\mathrm{DLA}}\right)$ decreases significantly with time between $z \sim 3.5$ and $z=2.2$.

Several explanations for the steepening of $f\left(N_{\mathrm{HI}}, \chi\right)$ have been discussed in the literature, including conversion from atomic to molecular hydrogen (Schaye 2001; Zwaan \& Prochaska 2006), small-scale turbulence, or stellar feedback (Erkal et al. 2012). Selection effects such as dust-reddening (e.g. Vladilo \& Péroux 2005) could also alter the slope of $f\left(N_{\mathrm{HI}}, \chi\right)$ in magnitude-limited surveys. However, the slope of the frequency distribution itself has not yet been well constrained at the highcolumn-density end due to fewer statistics. Similarly, the evolution of $\Omega_{\mathrm{g}}^{\mathrm{DLA}}$ has long been discussed in the literature. Values at $z \sim 1$ (Rao et al. 2006) have been considered uncomfortably 
high when compared to that at $z=0$ from Zwaan et al. (2005) and $z \sim 2$ from Prochaska et al. (2005). However, N09 corrected the value at $z \sim 2$ upwards. Since then, the value at $z=0$ has also been corrected upwards by Braun (2012) and could indicate a flatter evolution over $0<z<2$.

In this letter we present a search for DLAs in quasars observed in the course of the Baryonic Oscillation Spectroscopic Survey (BOSS, Dawson et al. 2012), one of the legacy surveys in the third stage of the SDSS (Eisenstein et al. 2011). We use the same formalism as described in N09 and adopt a $\Lambda$ CDM cosmology with $\Omega_{\Lambda}=0.73, \Omega_{\mathrm{m}}=0.27$, and $H_{0}=70 \mathrm{~km} \mathrm{~s}^{-1} \mathrm{Mpc}^{-1}$ (Komatsu et al. 2011).

\section{Method}

BOSS is a five-year programme using improved spectrographs (Smee et al. 2012) on the SDSS telescope (Gunn et al. 2006) to obtain spectra of 1.5 million galaxies and over $150000 z>$ 2.15 quasars reaching up to $1 \mathrm{mag}$ deeper than SDSS-II. The survey is mainly designed to measure the characteristic scale imprinted by baryon acoustic oscillations (BAOs) in the early Universe from the spatial distribution of luminous galaxies at $z \sim 0.7$ and the large-scale correlation of $\mathrm{H}_{\mathrm{I}}$ absorption lines in the intergalactic medium at $z \sim 2.5$ (Dawson et al. 2012). BOSS uses the same imaging data as in SDSS-I and II with an extension in the south galactic cap (see Aihara et al. 2011). The SDSS-DR9 (Ahn et al. 2012) makes publicly available the spectra of 87822 quasars over an area of $3275 \mathrm{deg}^{2}, 65205$ having $z>2$ (Pâris et al. 2012). The quasar target selection is described in Ross et al. (2012; see also Bovy et al. 2011).

\subsection{Detection of DLAs}

Intervening DLAs were automatically searched for in quasar spectra following the method described in N09. We briefly summarise here the main steps. For the purpose of collecting the largest number of DLAs ${ }^{1}$, we search the full line-of-sight to each quasar starting where the spectral signal-to-noise ratio per pixel reaches two (defining $z_{\text {min }}$ ) and up to the quasar redshift. We avoid sightlines with broad absorption lines of balnicity index BI > $1000 \mathrm{~km} \mathrm{~s}^{-1}$ (Pâris et al. 2012).

The quasar continuum is modelled over the Ly- $\alpha$ forest by fitting a modified power-law with a smoothly changing index plus Moffat profiles on top of the emission lines. Whenever the Ly- $\alpha$ emission line is severely absorbed (>30\%), we use the predicted unabsorbed emission from principal component analysis (see Pâris et al. 2011) as a proxy for the true Ly- $\alpha$ emission before fitting the continuum. We then use the median continuumto-noise ratio (CNR) as an estimate of the quality of the spectrum, independent of the presence of a DLA. Spectra with median CNR $<2$ over the Ly- $\alpha$ forest were not further considered.

Damped absorption lines are recognised through their characteristic shape by correlating the data against synthetic profiles of increasing column density. In short, $(N(\mathrm{H} \mathrm{I}), z)$ pairs with Spearman's correlation above 0.5 (and significance $>3 \sigma$ ) are recorded. To constrain the strength of the absorption, we also require that the absorbed flux is consistent with the presence of a DLA combined with possible Ly- $\alpha$ forest absorptions. The pairs are then grouped into individual DLA candidates (a gap of $>1000 \mathrm{~km} \mathrm{~s}^{-1}$ indicates separate absorption systems), and the

\footnotetext{
1 DLAs are contaminants for the study of the Ly- $\alpha$ forest correlation function (Font-Ribera \& Miralda-Escudé 2012).
}

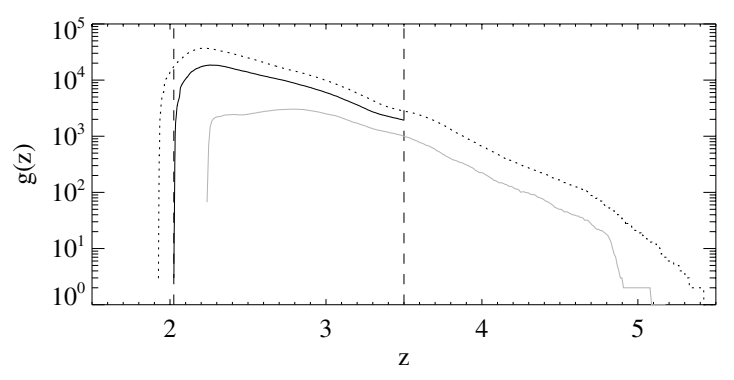

Fig. 1. Redshift sensitivity function $g(z)$ of our full DR9 sample (dotted) and statistical sample (black) compared to that of DR7 (N09, grey).

first guess for $N(\mathrm{HI})$ is taken from the pair with the highest correlation. The DLA redshift measurement is then improved whenever possible by cross-correlating the quasar spectrum on the red side of the Lyman- $\alpha$ emission line with a mask representing metal absorption lines. Finally, $N(\mathrm{HI})$ is obtained by fitting a Voigt profile to the damped Lyman- $\alpha$ line.

This approach provides us with an overall sample of 12081 DLA candidates with $\log N(\mathrm{H} \mathrm{I}) \geq 20$, out of which 6839 have $\log N(\mathrm{HI}) \geq 20.3$ (Table 3 ). We also provide values of (or limits on) the equivalent widths of associated metal lines redwards of the Ly- $\alpha$ emission line.

\subsection{Statistical sample}

We subsequently define a statistical sub-sample that is used to derive the $N(\mathrm{HI})$ distribution function and the integrated cosmological mass density of neutral gas. First of all, we conservatively reject all quasars with even moderate balnicity $(\mathrm{BI}>0$ or flagged visually, Pâris et al. 2012) and apply a more stringent threshold to the data quality, keeping only spectra with CNR $>3$. We then restrict the redshift range as follows: i) N09 showed that the presence of a DLA near the blue end of the spectrum can bias the definition of $z_{\min }$ and proposed an application of a systematic $10000 \mathrm{~km} \mathrm{~s}^{-1}$ velocity shift to $z_{\min }$ that we also apply here; ii) we consider only the region between $3000 \mathrm{~km} \mathrm{~s}^{-1}$ redwards of the Ly- $\beta$ emission line and $5000 \mathrm{~km} \mathrm{~s}^{-1}$ bluewards of the Ly- $\alpha$ emission line. The first cut ensures that we consider only the Ly- $\alpha$ forest and avoid the Ly- $\beta+\mathrm{O}$ vi region where associated broad O vi absorption can occur (even if no broad C IV absorption is seen) and be mistaken as DLAs. The second cut avoids DLAs located in the vicinity of the quasar (e.g. Ellison et al. 2002). Finally, we restrict our study to the range $z \in[2,3.5]$. This avoids the very blue end of the spectra (below $3650 \AA$ ) where reduction problems have been identified (Pâris et al. 2012). We set the upper limit because the increasing density of the Ly- $\alpha$ forest can introduce a significant fraction of false DLA identifications owing to strong blending of the Ly- $\alpha$ forest lines at the SDSS resolution (Rafelski et al. 2012). The value of 3.5 corresponds to the redshift out to which this systematic can be reliably estimated based on an analysis of mock spectra.

These cuts leave us with 37503 lines-of-sight bearing 5428 systems with $\log N(\mathrm{H} \mathrm{I}) \geq 20$ (3408 bona-fide DLAs with $\log N(\mathrm{HI}) \geq 20.3)$. We present in Fig. 1 the sensitivity functions $g(z)$ (i.e. the number of lines-of-sight covering a given redshift) for the full and statistical samples. Our statistical DR9 sample is more than three times larger than the overall DR7 sample and it also extends to lower redshifts, thanks to the improved blue coverage of the SDSS spectrograph. The total absorption path length probed by our statistical sample over $z=2-3.5$ is $\Delta \chi \approx 45000$ with an average redshift $\langle z\rangle=2.5$. 
P. Noterdaeme et al.: Distribution and evolution of neutral gas at $2<z<3.5$ from SDSS DR9
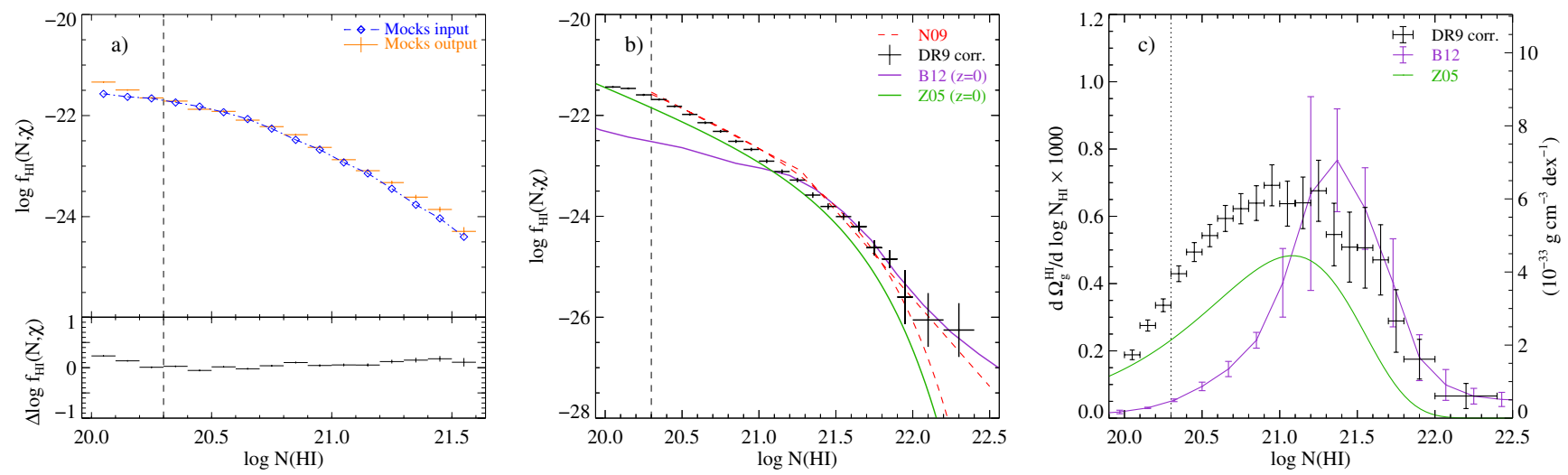

Fig. 2. Column density distribution functions from synthetic (left) and real data (centre) at $\langle z\rangle=2.5$. Horizontal bars represent the bin over which $f\left(N_{\mathrm{HI}}, \chi\right)$ is calculated and vertical error bars represent Poissonian uncertainty. The difference between output and input mock distributions is shown at the bottom of panel a). The double power-law and $\Gamma$-function fits to the DR7 distribution $(\mathrm{N} 09,\langle z\rangle=2.9)$ are shown as red dashed lines. $f\left(N_{\mathrm{HI}}, \chi\right)(z=0)$ are taken from Braun (2012, purple) and Zwaan et al. (2005, green). Right: the contribution of DLAs in a given $N(\mathrm{HI})$ range to the total mass census of neutral gas. DR9 values are corrected for systematics.

\subsection{Mocks}

The BOSS collaboration is constantly developing mocks that simulate the Lyman- $\alpha$ forest seen towards BOSS quasars (e.g. Font-Ribera et al. 2012). While mocks were principally designed for BAO studies, the important point for this study is that simulated spectra are produced with the same noise and flux distributions as in the actual DR9 data (Bailey et al., in prep.). In addition, DLAs have been introduced to those mocks with a known distribution (Font-Ribera \& Miralda-Escudé 2012). We also applied our DLA-searching algorithm to 33 realisations of 3861 mocks representative of the DR9 data with the same cuts as in real data. From this exercise, the completeness and purity ( 1 minus the fraction of false identifications) in the statistical sample are both found to be above $95 \%$ for $\log N(\mathrm{HI}) \geq 20.3$ (and higher when restricting to higher $N(\mathrm{H} \mathrm{I})$ systems). Overall, the automatic procedure systematically overestimates $N(\mathrm{H}$ I) by 0.03 dex. This is much lower than the dispersion ( 0.20 dex $)$ that corresponds to the typical $1 \sigma$ error on $\log N(\mathrm{H} \mathrm{I})$.

\section{The column density distribution at $\langle z\rangle=2.5$}

In Fig. 2a, we compare the simulated input distribution of $\mathrm{H}_{\mathrm{I}}$ column densities (in the range $N(\mathrm{HI})=10^{20}-4 \times 10^{21} \mathrm{~cm}^{-2}$ ) at $z=2-3$ with the one recovered from mocks by our procedure over the same redshift range. We can see that the overall agreement is excellent, although our procedure slightly overestimates $f\left(N_{\mathrm{HI}}, \chi\right)$, particularly at the low column density end. We use the difference between the input and output distributions as the correction to apply to the observed distribution from real data.

To ascertain the properties of the high-column-density end of $f\left(N_{\mathrm{HI}}, \chi\right)$, where statistics are much smaller, we have visually checked all DLA candidates with $\log N(\mathrm{HI}) \geq 21.6$. In this regime, blind correction using mocks could be more uncertain since the corresponding $\mathrm{H}_{\text {I }}$ fits are only based on Ly- $\alpha$, while metals are systematically detected in the real data. Indeed, we found a few cases where two closely-spaced DLAs were mistaken for a higher column density one. Disentangling such blends was possible thanks to the presence of metal lines. For each DLA candidate with $\log N(\mathrm{HI}) \geq 21$.6, the absorption profile was carefully refitted manually, improving the continuum determination and using metal lines to determine a precise redshift of the absorber. The resulting $f\left(N_{\mathrm{HI}}, \chi\right)$ at $z=2-3$ is shown in Fig. $2 b$ with values given in Table 1 . It is apparent that the
Table 1. $N(\mathrm{H}$ I) distribution function at $\langle z\rangle=2.5$.

\begin{tabular}{lccc}
\hline \hline $\log N(\mathrm{HI})$ & $\log f\left(N_{\mathrm{HI}}, \chi\right)$ & $\log f\left(N_{\mathrm{HI}}, \chi\right)_{\text {corr. }}{ }^{a}$ & $\sigma\left(\log f\left(N_{\mathrm{HI}}, \chi\right)\right)^{b}$ \\
\hline$[20.00,20.10[$ & -21.20 & -21.44 & 0.02 \\
{$[20.10,20.20[$} & -21.37 & -21.47 & 0.02 \\
{$[20.20,20.30[$} & -21.55 & -21.59 & 0.02 \\
{$[20.30,20.40[$} & -21.66 & -21.68 & 0.02 \\
{$[20.40,20.50[$} & -21.81 & -21.82 & 0.02 \\
{$[20.50,20.60[$} & -21.97 & -21.98 & 0.02 \\
{$[20.60,20.70[$} & -22.13 & -22.14 & 0.03 \\
{$[20.70,20.80[$} & -22.30 & -22.32 & 0.03 \\
{$[20.80,20.90[$} & -22.49 & -22.51 & 0.03 \\
{$[20.90,21.00[$} & -22.63 & -22.67 & 0.03 \\
{$[21.00,21.10[$} & -22.85 & -22.91 & 0.04 \\
{$[21.10,21.20[$} & -23.04 & -23.11 & 0.04 \\
{$[21.20,21.30[$} & -23.19 & -23.28 & 0.05 \\
{$[21.30,21.40[$} & -23.46 & -23.58 & 0.06 \\
{$[21.40,21.50[$} & -23.66 & -23.81 & 0.07 \\
{$[21.50,21.60[$} & -23.83 & -24.01 & 0.08 \\
{$[21.60,21.70[$} & -24.20 & -24.20 & 0.08 \\
{$[21.70,21.80[$} & -24.62 & -24.62 & 0.12 \\
{$[21.80,21.90[$} & -24.85 & -24.85 & 0.18 \\
{$[21.90,22.00[$} & -25.60 & -25.60 & 0.53 \\
{$[22.00,22.20[$} & -26.05 & -26.05 & 0.53 \\
{$[22.20,22.40[$} & -26.25 & -26.25 & 0.53 \\
\hline
\end{tabular}

Notes. ${ }^{(a)}$ Corrected for systematics. ${ }^{(b)}$ Poissonian errors.

distribution extends beyond $10^{22} \mathrm{~cm}^{-2}$ with five systems with $\log N(\mathrm{HI}) \geq 22$ in the statistical sample (8 in the full sample). Extrapolating this function, we might expect to detect DLAs reaching $\log N(\mathrm{HI})=23$ at the completion of BOSS.

Following N09, we measure the total amount of neutral gas in DLAs at $\langle z\rangle=2.5$ to be $\Omega_{\mathrm{g}}^{\mathrm{DLA}} \approx 10^{-3}$. Figure $2 \mathrm{c}$ represents the contribution to the total amount of neutral gas as a function of $N(\mathrm{H} \mathrm{I})$. We confirm the N09 result that the largest contribution comes from systems with $N(\mathrm{H} \mathrm{I}) \sim 10^{21} \mathrm{~cm}^{-2}$. However, it is interesting that the systems with $N(\mathrm{H} \mathrm{I})$ in excess of $5 \times 10^{21} \mathrm{~cm}^{-2}$ contribute a non-negligible fraction of $\Omega_{\mathrm{g}}^{\mathrm{DLA}}(\sim 10 \%)$, although they are rarely represented in most surveys.

\section{Cosmological mass density of neutral gas}

Figure 3 (see also Table 2) shows the evolution of the cosmological mass density in DLAs as a function of redshift. Using mock 


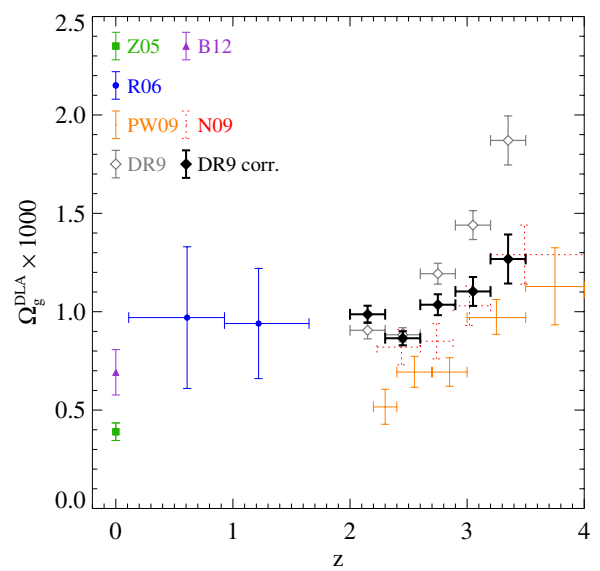

Fig. 3. Cosmological mass density of neutral gas in DLAs as a function of redshift (Z05: Zwaan et al. 2005; B12: Braun 2012; R06: Rao et al. 2006; PW09: Prochaska \& Wolfe 2009; DR9: this work).

Table 2. $\Omega_{\mathrm{g}}^{\text {DLA }}$ and DLA incidence (dN/dz) in different redshift bins.

\begin{tabular}{lccccc}
\hline \hline$z$ & $2.0-2.3$ & $2.3-2.6$ & $2.6-2.9$ & $2.9-3.2$ & $3.2-3.5$ \\
$\Delta z$ & 3690 & 4509 & 2867 & 1620 & 769 \\
$\Delta \chi^{*}$ & 11625 & 14841 & 9900 & 5834 & 2883 \\
$10^{3} \Omega_{\mathrm{g}}^{\text {DLA } \dagger}$ & $0.91 / 0.99$ & $0.88 / 0.87$ & $1.19 / 1.04$ & $1.44 / 1.10$ & $1.87 / 1.27$ \\
$10^{3} \sigma\left(\Omega_{\mathrm{g}}^{\text {DLA }}\right)^{\ddagger}$ & 0.05 & 0.04 & 0.05 & 0.08 & 0.13 \\
$\mathrm{~d} N / \mathrm{d} z^{\dagger}$ & $0.19 / 0.20$ & $0.21 / 0.20$ & $0.29 / 0.25$ & $0.36 / 0.29$ & $0.48 / 0.36$ \\
\hline
\end{tabular}

Notes. ${ }^{(*)}$ Total absorption pathlength (see Lanzetta et al. 1991). (†) Direct values/corrected for systematics. ${ }^{(\ddagger)}$ Statistical uncertainty.

spectra, we estimate a correction for systematics (over/underestimate of $N(\mathrm{HI})$, incompleteness, and contribution of false positives) as a function of redshift. At high redshift, the correction is mostly because of $N(\mathrm{HI})$ overestimation caused by the denser Ly- $\alpha$ forest together with increasing false positive identifications. At $z<2.3$, the correction is upwards owing to higher incompleteness and a slight underestimation of $N(\mathrm{HI})$. We note that the zero-point photometric calibration can be in error by about $5 \%$ below $4000 \AA$ (Pâris et al. 2012), which could affect the detection of DLAs and $N(\mathrm{H} \mathrm{I})$-measurements in different ways in mocks and real data. This problem will be addressed in forthcoming versions of the pipeline (Bolton et al. 2012).

We observe a decrease in $\Omega_{\mathrm{g}}^{\mathrm{DLA}}$ from $z=3.5$ to $z=2.3$ as in N09 and Prochaska \& Wolfe (2009), although with higher values at $z<3.2$. This can be explained by the $\sim 10 \%$ contribution of very large column density systems in DR9 and better knowledge of systematics. It is unclear which value of $\Omega_{\mathrm{g}}^{\mathrm{DLA}}$ should be used at $z=0$. The measurement by Braun (2012) is based on only three galaxies, and although the high-column density end of $f\left(N_{\mathrm{HI}}, \chi\right)$ seems to be well constrained, this may not be true at low $N(\mathrm{HI}$ ) (Zwaan et al. 2005). Measurements at $z \sim 1$ (Rao et al. 2006) are still indirect and, while direct searches for DLAs at low redshift are possible (Meiring et al. 2011), they are still quite limited in terms of sample size. It appears that systematics are a larger source of error than statistical uncertainties across most of the redshift range. Keeping this in mind, we can still conclude that $\Omega_{\mathrm{g}}^{\mathrm{DLA}}$ evolves only mildly over the past $12 \mathrm{Gyr}$.

\section{Conclusion}

We have presented the first results of our ongoing survey for DLAs in the SDSS-III BOSS, Data Release 9. This represents by far the largest sample of DLAs to date (with $\sim 12000$ systems with $\log N(\mathrm{HI}) \geq 20$ ) and should allow numerous follow-up studies. We expect the sample to be increased by a factor larger than two at the completion of BOSS. Using a well defined subsample, and controlling systematics (which dominate the error budget) with synthetic spectra, we derive the H I column density distribution at $\langle z\rangle=2.5$ in the range $10^{20}-2 \times 10^{22} \mathrm{~cm}^{-2}$ and characterised the evolution of the cosmological mass density of neutral gas in DLAs at $2<z<3.5$. This study should help to constrain models of galaxy formation and evolution by measuring the amount of neutral gas immediately available to fuel star formation through cosmic history.

Acknowledgements. We thank the anonymous referee for helpful comments and suggestions. The French participation group to SDSS-III was supported by the Agence Nationale de la Recherche under grant ANR-08-BLAN0222. Funding for SDSS-III has been provided by the Alfred P. Sloan Foundation, the Participating Institutions, the National Science Foundation, and the US Department of Energy Office of Science. The SDSS-III web site is http://www.sdss3.org/. SDSS-III is managed by the Astrophysical Research Consortium for the Participating Institutions of the SDSS-III Collaboration including the University of Arizona, the Brazilian Participation Group, Brookhaven National Laboratory, University of Cambridge, Carnegie Mellon University, University of Florida, the French Participation Group, the German Participation Group, Harvard University, the Instituto de Astrofísica de Canarias, the Michigan State/Notre Dame/JINA Participation Group, Johns Hopkins University, Lawrence Berkeley National Laboratory, Max Planck Institute for Astrophysics, Max Planck Institute for Extraterrestrial Physics, New Mexico State University, New York University, Ohio State University, Pennsylvania State University, University of Portsmouth, Princeton University, the Spanish Participation Group, University of Tokyo, University of Utah, Vanderbilt University, University of Virginia, University of Washington, and Yale University.

\section{References}

Ahn, C. P., Alexandroff, R., Allende Prieto, C., et al. 2012, ApJS, accepted [arXiv: 1207.7137]

Aihara, H., Allende Prieto, C., An, D., et al. 2011, ApJS, 193, 29

Bolton, A. S., Schlegel, D. J., Aubourg, E., et al. 2012, AJ, accepted [arXiv: 1207.7326]

Bovy, J., Hennawi, J. F., Hogg, D. W., et al. 2011, ApJ, 729, 141

Braun, R. 2012, ApJ, 749, 87

Dawson, K. S., Schlegel, D. J., Ahn, C. P., et al. 2012, AJ, submitted [arXiv: 1208.0022]

Eisenstein, D. J., Weinberg, D. H., Agol, E., et al. 2011, AJ, 142, 72

Ellison, S. L., Yan, L., Hook, I. M., et al. 2002, A\&A, 383, 91

Erkal, D., Gnedin, N. Y., \& Kravtsov, A. V. 2012, ApJ, submitted [arXiv: 1201.3653]

Font-Ribera, A., \& Miralda-Escudé, J. 2012, J. Cosmol. Astropart. Phys., 7, 28 Font-Ribera, A., McDonald, P., \& Miralda-Escudé, J. 2012, J. Cosmol. Astropart. Phys., 1, 1

Gunn, J. E., Siegmund, W. A., Mannery, E. J., et al. 2006, AJ, 131, 2332

Komatsu, E., Smith, K. M., Dunkley, J., et al. 2011, ApJS, 192, 18

Lah, P., Chengalur, J. N., Briggs, F. H., et al. 2007, MNRAS, 376, 1357

Lanzetta, K. M., McMahon, R. G., Wolfe, A. M., et al. 1991, ApJS, 77, 1

Meiring, J. D., Tripp, T. M., Prochaska, J. X., et al. 2011, ApJ, 732, 35

Noterdaeme, P., Petitjean, P., Ledoux, C., \& Srianand, R. 2009, A\&A, 505, 1087

Pâris, I., Petitjean, P., Rollinde, E., et al. 2011, A\&A, 530, A50

Pâris, I., Petitjean, P., Aubourg, E., et al. 2012, A\&A, in press, DOI: $10.1051 / 0004-6361 / 201220142$

Prochaska, J. X., \& Wolfe, A. M. 2009, ApJ, 696, 1543

Prochaska, J. X., Herbert-Fort, S., \& Wolfe, A. M. 2005, ApJ, 635, 123

Prochaska, J. X., Sheffer, Y., Perley, D. A., et al. 2009, ApJ, 691, L27

Rafelski, M., Wolfe, A. M., Prochaska, J. X., Neeleman, M., \& Mendez, A. J. 2012, ApJ, 755, 89

Rao, S. M., Turnshek, D. A., \& Nestor, D. B. 2006, ApJ, 636, 610

Ross, N. P., Myers, A. D., Sheldon, E. S., et al. 2012, ApJS, 199, 3

Schaye, J. 2001, ApJ, 562, L95

Smee, S., Gunn, J. E., Uomoto, A., et al. 2012, AJ, submitted [arXiv: 1208.2233]

Vladilo, G., \& Péroux, C. 2005, A\&A, 444, 461

Wolfe, A. M., Gawiser, E., \& Prochaska, J. X. 2005, ARA\&A, 43, 861

York, D. G., Adelman, J., Anderson, Jr., J. E., et al. 2000, AJ, 120, 1579

Zwaan, M. A., \& Prochaska, J. X. 2006, ApJ, 643, 675

Zwaan, M. A., van der Hulst, J. M., Briggs, F. H., Verheijen, M. A. W., \& Ryan-Weber, E. V. 2005, MNRAS, 364, 1467 